\title{
Shrimp-Allergic Patients in a Multi-Food Oral Immunotherapy Trial
}

\author{
Carla McGuire Davis ${ }^{1}$, Diem-Tran Nguyen ${ }^{1}$, Sayantani Sindher ${ }^{2}$, R. Chinthrajah ${ }^{3}$, and \\ Kari Nadeau ${ }^{2}$ \\ ${ }^{1}$ Baylor College of Medicine \\ ${ }^{2}$ Stanford University School of Medicine \\ ${ }^{3}$ Stanford University
}

July 12, 2021

Title :

Shrimp-Allergic Patients in a Multi-Food Oral Immunotherapy Trial

Authors :

Diem-Tran I. Nguyen MD ${ }^{1}$, Sayantani B. Sindher $\mathrm{MD}^{2,3}$, R. Sharon Chinthrajah MD ${ }^{2,3}$, Kari Nadeau MD $\mathrm{PhD}^{2,3}$, Carla M. Davis $\mathrm{MD}^{1,4}$

${ }^{1}$ Department of Pediatrics, Baylor College of Medicine, Houston, TX, United States

${ }^{2}$ Sean N. Parker Center for Allergy and Asthma Research, Stanford University, Palo Alto, CA, USA

${ }^{3}$ Division of Pulmonary, Allergy and Critical Care Medicine, Dept of Medicine, Stanford, CA, USA.

${ }^{4}$ Section of Immunology, Allergy and Retrovirology, Baylor College of Medicine and Texas Children's Hospital, Houston, TX, United States

Corresponding Author:

Carla M. Davis

carlad@bcm.edu

Word Count: 978

To the Editor:

Shellfish allergy is one of the most common food allergies in the United States accounting for approximately $25 \%$ of adulthood and $20 \%$ of childhood food allergies (FA) ${ }^{1,2}$ Of the different types of shellfish, shrimp are considered the most allergenic. The prevalence of shellish allergy in children is substantial at $1.3 \%$ and may result in a greater prevalence in the adult population (3\%) given that shellfish allergies have a low rate of spontaneous resolution. ${ }^{2,3}$

Shrimp allergy (SA) is a leading cause of severe food reactions and results in high rates of healthcare usage. ${ }^{4}$ Nearly $50 \%$ of patients with SA experience at least one lifetime food allergy related Emergency Department visit, yet only $42 \%$ of adults and $61 \%$ of children with SA reported having a physician confirmed diagnosis. ${ }^{1,2}$ The lack of physician confirmation of SA is concerning given the potentially life threatening consequences of accidental exposure. ${ }^{5}$ Currently, there is no cure and the only management strategies are 
avoidance and treatment for severe reactions with epinephrine. ${ }^{6}$ However, avoidance can be difficult due to the high incidence of cross-contamination, requiring strict dietary limitations.

Oral immunotherapy (OIT) has emerged as a promising treatment for FA. In OIT, patients ingest increasing doses of the allergenic food with the goal of achieving desensitization so that reactions are less severe. Once a maintenance dose is achieved, the allergen needs to be regularly ingested to preserve the desensitized state. Although OIT has been recently approved by the FDA for peanut allergies, there has been little data in shrimp allergic patients. In this case-series, we discuss a subset of three patients who received shrimp OIT as part of a phase II, multi-food, omalizumab-facilitated OIT clinical trial.

Multi-food allergic patients were recruited to a multi-site clinical trial between January 1 and November 30, 2016. Full details of trial design, inclusion criteria, and exclusion criteria have been previously reported. ${ }^{7}$ Patients initially underwent testing with skin prick testing (SPT), specific IgE testing, and doubleblind placebo-controlled food challenge (DBPCFC) to confirm their allergy to their culprit foods. To be included, patients were required to have a positive $\mathrm{SPT}$ of $>6 \mathrm{~mm}$ wheal diameter, specific $\operatorname{IgE}>0.35 \mathrm{kU} / \mathrm{L}$, a total $\operatorname{IgE}<2,000 \mathrm{kU} / \mathrm{L}$, and a clinical reaction with DBPCFCs at $<125 \mathrm{mg}$ dose.

Patients enrolled in this clinical trial received $0.016 \mathrm{mg} / \mathrm{kg}(\mathrm{IU} / \mathrm{mL})$ omalizumab per month or $0.008 \mathrm{mg} / \mathrm{kg}$ $\left(\mathrm{IU} / \mathrm{mL}\right.$ ) every two weeks (based on asthma dosing guidelines) ${ }^{7}$ from week 1-16. At week 8, multi-food OIT was started and escalated under an investigator-supervised multi-OIT up-dosing regimen to reach a maintenance dose of $>1 \mathrm{~g}$ of each allergen. Participants who reached maintenance by week 28-29 were randomized and received week $30 \mathrm{DBPCFC}$ to assess desensitization to the allergenic foods. Patients were then randomized to one of three arms: high dose maintenance $(1000 \mathrm{mg})$, low dose maintenance $(300 \mathrm{mg})$, or placebo $(0 \mathrm{mg})$. This randomized dose was dispensed at the last week 30 DBPCFC and consumed until week 36. At week 36, DBPCFC was repeated to assess sustained unresponsiveness with differing daily doses of protein.

A total of 70 patients were enrolled, with three found to have SA. Their demographic data and baseline characteristics are detailed in Table 1. All three patients also had asthma, allergic rhinitis, and atopic dermatitis. Each had a convincing clinical history, elevated total IgE, and positive SPT to a mixture of white, brown, and pink shrimp extract from Greer. The diagnosis was confirmed by a reaction during DBPCFC withLitopenaeus setiferus shrimp flour that was manufactured at a Good Manufacturing Practice facility at Stanford University.

Clinical outcomes and adverse events are detailed in Table 2. All 3 patients tolerated dose escalation without serious adverse events or epinephrine requirement, were able to achieve maintenance dose, and did not have an allergic reaction at the Week 30 DBPCFC. Patient A was randomized to the placebo treatment arm while the other two patients were randomized to the $300 \mathrm{mg}$ maintenance OIT arm. At Week 36, Patient A and Patient B had sustained unresponsiveness to $12,000 \mathrm{mg}$ of shrimp extract. Patient $\mathrm{C}$ did not follow-up for assessment.

It is encouraging that all 3 shrimp allergic patients in this multi-food OIT clinical trial were able to reach maintenance dose OIT $(>1 \mathrm{~g})$, and 2 out of 3 had no reaction with the $12 \mathrm{~g}$ DBPCFC dose at Week 30 . These results suggest that OIT is a potentially efficacious treatment for SA and warrants further study. There is little data on the optimal shrimp allergen product, dose escalation regimen, and adjunct therapies such as omalizumab to achieve desensitization.

There are several known target allergens that contribute to SA. The first major allergen is tropomyosin, a heat-stable, actin-binding protein found in both muscle and non-muscle cells. Tropomyosin has been implicated as the source of significant cross-reactivity between species of mollusks, crustaceans, and non-shellfish such as cockroaches and mites. ${ }^{8,9}$ Other shrimp allergens that have been identified include arginine kinase, myosin light chain, sarcoplasmic calcium-binding protein, hemocyanin, and troponin C. ${ }^{8,9}$ It is possible that patients with allergies to different shrimp components may have varied responses to OIT, and thus additional research is necessary to determine which patient subgroups are most likely to benefit from shrimp OIT. 
Our case series is limited by small sample size, with only three patients receiving shrimp OIT and two following up at week 36. Although all patients appeared to develop short-term tolerance by week 30 , it is unclear how durable this response would be with long-term follow up. Furthermore, there are risks associated with OIT.

$\mathrm{SA}$ is a common and serious food allergy that is underdiagnosed and often lifelong. There are currently no effective treatments other than strict avoidance, which can be difficult to achieve and lead to poor quality of life. Our case series presents initial evidence suggesting that shrimp OIT may be an effective strategy of addressing grave reactions faced by SA patients. Larger studies need to be performed to validate these findings.

\section{References :}

1. Gupta, R. S. et al.Prevalence and Severity of Food Allergies Among US Adults. JAMA Netw Open 2, e185630 (2019).

2. Wang, H. T., Warren, C. M., Gupta, R. S. \& Davis, C. M. Prevalence and Characteristics of Shellfish Allergy in the Pediatric Population of the United States. J. Allergy Clin. Immunol. Pract. 8, 1359-1370.e2 (2020).

3. Zotova, V. et al.Low resolution rates of seafood allergy. J. Allergy Clin. Immunol. Pract. 7, 690-692 (2019).

4. Ross, M. P. et al.Analysis of food-allergic and anaphylactic events in the National Electronic Injury Surveillance System. J. Allergy Clin. Immunol.121, 166-171 (2008).

5. Tuano, K. T. S. et al. Improved diagnostic clarity in shrimp allergic non-dust-mite sensitized patients. Allergy Asthma Proc. 39, 377-383 (2018).

6. Davis CM, Gupta RS, Aktas ON, Diaz V, Kamath SD, Lopata AL. Clinical Management of Seafood Allergy. J Allergy Clin Immunol Pract. 2020 Jan;8(1):37-44.

7. Andorf, S. et al. A Phase 2 Randomized Controlled Multisite Study Using Omalizumab-facilitated Rapid Desensitization to Test Continued Discontinued Dosing in Multifood Allergic Individuals.EClinicalMedicine 7, 27-38 (2019).

8. Faber, M. A. et al.Shellfish allergens: tropomyosin and beyond. Allergy 72, 842-848 (2017).

9. Ruethers T, Taki AC, Johnston EB, Nugraha R, Le TTK, Kalic T, McLean TR, Kamath SD, Lopata AL. Seafood allergy: A comprehensive review of fish and shellfish allergens. Mol Immunol. 2018 Aug;100:28-57.

\section{Hosted file}

Shrimp OIT Letter to Editor Tables - Submitted.docx available at https://authorea.com/users/ 425109/articles/529997-shrimp-allergic-patients-in-a-multi-food-oral-immunotherapy-trial 